\title{
Employment status, hours working, and gainful earnings after spinal cord injury: relationship with pain, prescription medications for pain, and nonprescription opioid use
}

\author{
James S. Krause ${ }^{1} \cdot$ Clara E. Dismuke-Greer ${ }^{1} \cdot$ Karla S. Reed $^{1} \cdot$ Chao $\mathrm{Li}^{1}$ \\ Received: 26 July 2019 / Revised: 25 September 2019 / Accepted: 18 October 2019 / Published online: 1 November 2019 \\ (c) The Author(s), under exclusive licence to International Spinal Cord Society 2019
}

\begin{abstract}
Study design Cross-sectional self-report assessment. Econometric modeling.

Objectives Identify the relationship of multiple pain indicators, prescription pain medication, nonprescription opioid use, and multiple indicators of quality employment among those with spinal cord injury (SCI).

Setting Data were collected at a medical university in the Southeastern United States (US).

Methods Participants included 4670 adults with traumatic SCI of at least one-year duration who were enrolled in a study of health and longevity. They were identified from three sources including a specialty hospital and two population-based state SCI surveillance systems. Econometric modeling was used for three outcome variables: employment status, hours per week spent working, and earnings.

Results Several pain parameters were significantly related to multiple employment outcomes. Prescription medication to treat pain was associated with lower odds of employment, fewer hours working, and lower conditional earnings. Nonprescription opioid use was only related to fewer hours working. Painful days, number of painful conditions, and pain intensity were all related to employment outcomes, but the pattern varied by outcome. The number of painful conditions was most consistently related to employment. Multiple demographic, injury, and educational factors were related to employment, with better outcomes among those with less severe SCI and greater educational achievements.

Conclusions The presence of significant pain and use of either prescription pain medications or the use of nonprescription opioids may have a significant adverse effect on both the probability of employment and quality of employment. Rehabilitation and vocational professionals should routinely assess pain and associated medications in vocational and career planning.
\end{abstract}

\section{Introduction}

Traumatic spinal cord injury (SCI) is a severe disabling condition associated with lifelong risk of secondary health conditions adversely affecting participation, including employment [1-4]. Estimates suggest as few as 35-40\% of persons with SCI are employed after injury [5-7], and retaining employment after SCI is difficult $[8,9]$. Beyond employment rates, the limited existing research on quality employment outcomes reveals lower earnings [10], decreased

James S. Krause

krause@musc.edu

1 College of Health Professions, Medical University of South Carolina, Charleston, SC, USA job satisfaction [11], and concerns about work intensity (hours employed) after SCI [12]. Furthermore, underemployment and low income are related to a greater risk of mortality $[13,14]$, highlighting the importance of the interrelationships between employment, health, and longevity.

Pain is a particularly debilitating secondary health condition faced by persons with SCI [15-17], with chronic pain of more than 12 weeks frequently reported [18]. Chronic pain after SCI has been shown to interfere with activity and participation (e.g., paid employment) [19] and has been identified as a barrier to earning potential [20-22]. A pharmacological approach is often used to treat pain after SCI [16, 23], with evidence showing polypharmacy is more prevalent among persons with SCI when compared with matched controls in the general population, which is associated with a greater risk of drug-related problems [24]. Specifically, misuse of prescription pain medications, including prescribed opioids, has 
been indicated in persons with SCI $[25,26]$, yielding the need for further research on prescription pain medication, opioids, and related consequences with SCI.

Pain and pain medication use could decrease the likelihood of employment after SCI [19], as well as work intensity and earnings for those who are employed. Nonmedical opioid use, either use of opioids that have not been prescribed or using prescription opioids in a manner different from which they were prescribed, may also present a barrier to quality employment. Despite this potential for pain and pharmacological treatments for pain to diminish employment outcomes, there has been a void of research to identify these relationships, with nearly all studies focusing solely on current employment rates [6, 21]. Identifying such relationships is vital, as it could impact decisions made by individuals with SCI and rehabilitation and medical professionals regarding the use of pharmacological treatments for pain after SCI.

Our purpose was to identify the relationship of multiple pain indicators (number of painful days in the last month, number of painful conditions, and average pain intensity), prescription pain medication, and use of nonprescription opioids with multiple indicators of quality employment, while controlling for demographic, injury, and educational characteristics. Quality employment includes employment outcomes beyond employment status alone. They may be objective indicators, as measured in the current study by earnings and work hours, or subjective, such as job satisfaction.

\section{Methods}

\section{Participants}

Institutional review board approval was obtained prior to data collection. Eligibility criteria at enrollment included: (1) adult, (2) traumatic SCI with residual deficits (noncomplete recovery consistent with American Spinal Injury Association Impairment Scale A-D), and (3) minimum of 12 months post-injury. The study participants were identified from five participant cohorts totaling 4670 participants. The first three cohorts were from a specialty hospital in the Southeastern United States and were first enrolled into a larger longitudinal study of health outcomes at one of three points in time: 1997-1998, 2007-2009, and 2014-2016. Although these three cohorts were first enrolled in longitudinal research and at different points in time, all data used in the current analysis were collected between 2011 and 2016. There were 2516 participants from these three cohorts (cohort $1=673$; cohort $2=1255$; cohort $3=588$ ).

The other two cohorts were recruited from populationbased state SCI surveillance registries in the Southeastern and Midwestern United States, which are inclusive of all civilians treated for acute SCI identified through state hospital discharge data using International Classification of Diseases-9, Clinical Modification codes of 806 and 952, following Centers for Disease Control guidelines [27]. The population-based cohorts were enrolled in 2012-2016 (Southeastern $n=1018$; Midwestern $n=1136$ ). After excluding those who could not be located, the overall response rate was $60.4 \%$. The response rate was higher for participants enrolled through the specialty hospital $(73.9 \%)$ as opposed to the surveillance systems (49.7\%).

\section{Procedures}

The data were collected between 2011 and 2016. Preliminary cover letters were sent to potential participants to alert them materials would be forthcoming, followed by the instrument package 4-6 weeks later. There were two follow-up mailings and a follow-up phone call. Participants were paid $\$ 50$ remuneration to promote response.

\section{Measures}

Demographic, injury, and educational variables included sex, race/ethnicity, age at injury onset, years since injury, injury severity, relationship status, and educational milestones. These variables were broken down into discrete categories for statistical analysis (see detail in tables). For instance, injury severity was broken into four groups, with one group comprised of all ambulatory participants and the remaining participants grouped according to the following injury levels: $\mathrm{C} 1-\mathrm{C} 4$ (reference group), C5-C8, and noncervical. Quality employment was defined as any indicator quantifying employment other than simply employment status or employment rates, specifically earnings and hours working per week in this study. Earnings were measured by asking individuals to indicate salary/earnings from their current or most recent post-injury job, with 10 response categories ranging from $<\$ 10,000$ to $\$ 150,000$ or more. Hours working per week were measured with a single item stated as, "What is the average number of hours per week you currently work?"

Pain indicators included the number of painful days within the past month, taken from the Behavioral Risk Factor Surveillance System [28]. The Pain Medication Questionnaire assessed the number of painful conditions [29, 30], and average pain intensity was taken from the Brief Pain Inventory [31]. Participants were asked how frequently they take prescription medication for pain, which was coded as never, occasionally, and daily (reference group). Use of nonprescription opioids was classified as yes/no, using the Alcohol, Smoking, and Substance Involvement Screening Test (ASSIST) developed by the World Health Organization [32, 33]. 


\section{Analyses}

Descriptive statistics were generated to summarize participant characteristics. The Chi-square statistic was used to test group differences. Econometric modeling was used to develop separate models for each outcome. Econometric modeling estimates multiple parameters using a two-part hurdle model. We differentiated between conditional and unconditional earnings and hours, as conditional variables are conditional upon being employed. Therefore, they were only calculated among those who are employed. In contrast, unconditional outcomes were calculated for all participants, allocating a score of 0 for those unemployed (i.e., 0 hours and 0 earnings).

The adjusted likelihood of employment was estimated using logit models. We examined the adjusted unconditional number of hours associated with each set of predictors using a two-part model with a Poisson family due to the number of hours being a finite count [34]. Marginal effects of each covariate can be interpreted directly. First, a binary choice model was estimated for the probability of observing a positive-versus-zero outcome. Then, conditional on a positive outcome for employment (i.e., employed), a generalized linear model (GLM) assuming a Poisson distribution was estimated for the number of hours. A standard GLM model was used to estimate the number of hours (employed only).

Adjusted unconditional earnings associated with each set of predictors were estimated using extended interval regression and adjusted conditional earnings (employed only) using standard interval regression. Interval regression takes into consideration the lower and upper limits of earnings for individuals who reported income $<\$ 10,000$ and those whose earnings were greater than the upper interval value. A generalization of censored regression, standard interval regression incorporates the minimum and maximum values of the earnings intervals, so information was not lost in estimation. When incorporating interval regression into a two-part model, intreg, was used to estimate unconditional earnings (employed and unemployed). This accounts for endogenous sample selection (i.e., employment status and measurement of unconditional estimates are not independent). Standard interval regression, intreg, was used for estimating conditional earnings (employed only).

\section{Results}

Table 1 summarizes the participant characteristics as a function of cohort. Of the 4670 participants, 4276 had complete data and were included in the econometric modeling.

\section{Regression}

\section{Employment status}

Injury severity, race/ethnicity, age at onset, years postinjury, relationship status, and educational milestones were all significantly related to employment status (Table 2). The highest odds of employment were observed among NonHispanic Whites $(\mathrm{OR}=3.41$ compared with non-Hispanic Blacks), among the three youngest age groups at onset, among those with 10 or more years post-injury, and those who were married or part of an unmarried couple $(\mathrm{OR}=$ 1.58) compared with those divorced/widowed/separated. The odds of employment improved with each successive level of educational attainment.

Those with $0-5$ painful days had 1.30 odds of employment relative to those with more than 20 painful days. Those with 6-20 painful days also had greater odds of employment $(\mathrm{OR}=1.27)$. Relative to those with 3-5 painful conditions, those with $0-1$ painful conditions $(\mathrm{OR}=1.81)$ and those with 2 painful conditions $(\mathrm{OR}=1.70)$ had higher odds of employment. Average pain was not significant. Relative to daily users, those who never used pain medication $(\mathrm{OR}=$ 2.11 ) and those who used pain medication occasionally $(\mathrm{OR}=1.51)$ had higher odds of employment. Use of nonprescription opioids was not significant.

\section{Conditional hours (employed only)}

When considering only employed participants (Table 3), compared with $\mathrm{C} 1-\mathrm{C} 4$, higher average hours per week were observed among C5-C8 (3.99), noncervical (6.30), and ambulatory SCI (11.03). Men reported 4.08 more average hours working compared with women. Participants who were married or part of an unmarried couple averaged 3.14 greater hours than those divorced/widowed/separated. Each of the younger groups based on age at onset reported greater hours than those in the oldest group at onset. The number of hours employed increased with each successive level of education compared with those who had the least education, ranging from $1.33-4.29$ greater hours.

Greater conditional hours were reported by those with average pain intensity of 2 or less (1.23). Greater hours were also observed by those with no use of nonprescription opioids (4.87) and those who either never used prescription medication (3.14) or used it occasionally but not daily (1.86).

\section{Unconditional hours (all participants)}

Participants with C5-C8 (2.29), noncervical (4.30), and ambulatory (10.75) had higher unconditional hours compared with C1-C4 injury severity (Table 4). Men reported 
Table 1 Bivariate comparison of the three cohorts on predictor variables

\begin{tabular}{|c|c|c|c|c|c|c|c|}
\hline & \multicolumn{2}{|c|}{ Midwestern } & \multicolumn{2}{|c|}{ Southeastern } & \multicolumn{2}{|c|}{ Clinical } & \multirow[t]{2}{*}{$p$-value } \\
\hline & $n$ & $\%$ & $n$ & $\%$ & $n$ & $\%$ & \\
\hline Injury severity & & & & & & & $<0.001$ \\
\hline $\mathrm{C} 1-\mathrm{C} 4$, nonambulatory & 57 & 5.1 & 60 & 6.1 & 266 & 10.6 & \\
\hline C5-C8, nonambulatory & 120 & 10.8 & 103 & 10.4 & 603 & 24.1 & \\
\hline Noncervical, nonambulatory & 201 & 18.1 & 166 & 16.8 & 844 & 33.7 & \\
\hline Ambulatory & 734 & 66.0 & 659 & 66.7 & 792 & 31.6 & \\
\hline Sex & & & & & & & 0.518 \\
\hline Female & 293 & 25.8 & 276 & 27.1 & 635 & 25.3 & \\
\hline Male & 843 & 74.2 & 742 & 72.9 & 1880 & 74.8 & \\
\hline Marital status & & & & & & & $<0.001$ \\
\hline $\begin{array}{l}\text { Divorced/widowed/ } \\
\text { separated }\end{array}$ & 223 & 19.9 & 279 & 27.8 & 619 & 24.8 & \\
\hline $\begin{array}{l}\text { Married/member of } \\
\text { unmarried couple }\end{array}$ & 616 & 55.1 & 470 & 46.9 & 1163 & 46.6 & \\
\hline Never married & 279 & 25.0 & 254 & 25.3 & 713 & 28.6 & \\
\hline Race & & & & & & & $<0.001$ \\
\hline Hispanic & 24 & 2.2 & 23 & 2.3 & 64 & 2.6 & \\
\hline Non-Hispanic Black & 34 & 3.1 & 348 & 35.3 & 498 & 20.4 & \\
\hline Non-Hispanic White & 985 & 90.0 & 589 & 59.7 & 1811 & 74.2 & \\
\hline Other & 51 & 4.7 & 27 & 2.7 & 69 & 2.8 & \\
\hline Age at onset & & & & & & & $<0.001$ \\
\hline$<30$ & 369 & 33.5 & 268 & 27.8 & 1183 & 47.2 & \\
\hline$\geq 30$ and $<40$ & 196 & 17.8 & 140 & 14.5 & 491 & 19.6 & \\
\hline$\geq 40$ and $<50$ & 192 & 17.4 & 179 & 18.6 & 388 & 15.5 & \\
\hline$\geq 50$ & 344 & 31.2 & 376 & 39.0 & 445 & 17.8 & \\
\hline Time since onset & & & & & & & $<0.001$ \\
\hline$<10$ & 518 & 54.6 & 640 & 82.7 & 1195 & 51.7 & \\
\hline$\geq 10$ and $\leq 19$ & 339 & 35.8 & 106 & 13.7 & 552 & 23.9 & \\
\hline$\geq 20$ & 91 & 9.6 & 28 & 3.6 & 565 & 24.4 & \\
\hline Education & & & & & & & $<0.001$ \\
\hline$\leq$ high school & 422 & 39.8 & 373 & 47.0 & 792 & 35.3 & \\
\hline 2-year degree/trade school & 339 & 32.0 & 274 & 34.6 & 657 & 29.3 & \\
\hline 4-year degree & 190 & 17.9 & 86 & 10.8 & 506 & 22.5 & \\
\hline Postgraduate & 109 & 10.3 & 60 & 7.6 & 290 & 12.9 & \\
\hline Painful days & & & & & & & $<0.001$ \\
\hline $0-5$ & 627 & 56.3 & 352 & 35.1 & 1374 & 55.1 & \\
\hline $6-20$ & 254 & 22.8 & 285 & 28.4 & 622 & 24.9 & \\
\hline$>20$ pain days & 233 & 20.9 & 365 & 36.4 & 498 & 20.0 & \\
\hline Painful conditions & & & & & & & $<0.001$ \\
\hline $0-1$ & 502 & 45.1 & 333 & 33.1 & 1130 & 45.3 & \\
\hline 2 & 308 & 27.7 & 258 & 25.7 & 656 & 26.3 & \\
\hline $3-5$ & 302 & 27.2 & 414 & 41.2 & 709 & 28.4 & \\
\hline Average pain intensity & & & & & & & $<0.001$ \\
\hline$\leq 2$ & 370 & 33.3 & 162 & 16.3 & 660 & 26.6 & \\
\hline $3-4$ & 325 & 29.3 & 200 & 20.1 & 644 & 26.0 & \\
\hline$\geq 5$ & 415 & 37.4 & 635 & 63.7 & 1175 & 47.4 & \\
\hline Pain medications & & & & & & & $<0.001$ \\
\hline Never use & 524 & 46.7 & 227 & 22.5 & 833 & 33.3 & \\
\hline Sometimes use & 231 & 20.6 & 235 & 23.3 & 596 & 23.8 & \\
\hline Daily use & 368 & 32.8 & 546 & 54.2 & 1076 & 43.0 & \\
\hline Nonprescription opioid use & & & & & & & 0.237 \\
\hline No & 1082 & 96.4 & 915 & 96.4 & 2416 & 97.3 & \\
\hline Yes & 40 & 3.6 & 34 & 3.6 & 67 & 2.7 & \\
\hline
\end{tabular}

1.73 higher average number of hours. Participants who were married or part of an unmarried couple averaged 3.30 greater hours than those divorced/widowed/separated. NonHispanic Whites (6.00) and other race (3.98) averaged greater hours than non-Hispanic Blacks. Those younger at injury reported more hours working. Compared with those who had no more than a high school certificate, each successive educational milestone was associated with greater unconditional hours, ranging from 3.39-7.61.

Greater unconditional hours were reported by participants with the fewest painful days (2.14) and those with either 0-1 painful conditions (2.97) or 2 painful conditions (3.38). Greater unconditional hours were reported by those with no use of nonprescription opioids (3.53), no prescription pain medication use (5.68), or occasional prescription pain medication use (3.25).

\section{Conditional earnings}

None of the pain parameters was significantly related to conditional earnings (Table 5). Several demographic and injury variables were related to conditional earnings. Higher earnings were reported among those with noncervical SCI $(\$ 14,329)$ and those who were ambulatory $(\$ 23,080)$ compared with C1-C4 injury severity. Higher conditional earnings were observed among men $(\$ 19,238)$, non-Hispanic Whites $(\$ 17,042)$, and those who were married or part of an unmarried couple $(\$ 7,481)$. Those in the age group of $40-49$ reported higher conditional earnings $(\$ 16,429)$ compared with those in the oldest group. Higher conditional earnings were observed with each successively higher educational milestone, with the greatest difference between those with postgraduate degrees compared with those with no more than a high school $(\$ 42,420)$.

\section{Unconditional earnings}

The pattern for demographic, injury, and educational factors was similar to that of conditional earnings, although the differences were magnified in many cases (Table 6). For instance, non-Hispanic Whites reported $\$ 47,258$ greater unconditional earnings compared with non-Hispanic Blacks. Those with less severe SCI reported substantially higher unconditional earnings compared with those with the most severe SCI (C1-C4), ranging from a low of $\$ 22,970$ for those with C5-C8 to a high of $\$ 64,995$ for those who were ambulatory. Compared with participants with no more than a high school certificate, those with increasing education levels reported higher unconditional earnings ranging from $\$ 24,545$ to $\$ 64,758$. 
Table 2 Logit model of employment status $(n=4276)$

\begin{tabular}{rlr}
\hline Odds ratio & $95 \% \quad p$-value \\
& confidence \\
& interval \\
\hline
\end{tabular}

Injury severity (ref: $\mathrm{C} 1-\mathrm{C} 4$, nonambulatory)

$\begin{array}{lrrrr}\text { C5-C8, nonambulatory } & 1.59 & 1.11 & 2.30 & 0.012 \\ \text { Noncervical, nonambulatory } & 2.10 & 1.48 & 2.98 & <0.001 \\ \text { Ambulatory } & 4.85 & 3.46 & 6.81 & <0.001 \\ \begin{array}{l}\text { Sex (ref: Female) } \\ \text { Male }\end{array} & 1.08 & 0.90 & 1.28 & 0.413\end{array}$

Marital status (ref: Divorced/widowed/separated)

$\begin{array}{lllll}\begin{array}{l}\text { Married/member of } \\ \text { unmarried couple }\end{array} & 1.58 & 1.29 & 1.93 & <0.001 \\ \end{array}$

$\begin{array}{lllll}\text { Never married } & 1.12 & 0.88 & 1.43 & 0.360\end{array}$

Race (ref: Non-Hispanic Black)

$\begin{array}{lllll}\begin{array}{l}\text { Non-Hispanic White } \\ \text { Other }\end{array} & 3.41 & 2.66 & 4.38 & <0.001 \\ \text { Age at onset (ref: } \geq 50) & 2.12 & 1.41 & 3.18 & <0.001 \\ <30 & & & & \\ \geq 30 \text { and }<40 & 3.17 & 2.55 & 3.95 & <0.001 \\ \geq 40 \text { and }<50 & 2.90 & 2.26 & 3.72 & <0.001 \\ \text { Time since onset (ref: }<10) & 2.16 & 1.67 & 2.80 & <0.001 \\ \geq 10 \text { and } \leq 19 & 1.34 & 1.11 & 1.62 & 0.003 \\ \geq 20 & 1.25 & 1.04 & 1.52 & 0.020\end{array}$

Education (ref: $\leq$ High School)

$\begin{array}{lllll}\text { 2-year degree/trade school } & 1.80 & 1.49 & 2.17 & <0.001 \\ \text { 4-year degree } & 3.05 & 2.47 & 3.77 & <0.001 \\ \text { Postgraduate } & 3.06 & 2.38 & 3.93 & <0.001 \\ \text { Painful days (ref: }>\text { 20 pain days) } & & & & \\ 0-5 & 1.30 & 1.01 & 1.69 & 0.045 \\ 6-20 & 1.27 & 1.00 & 1.61 & 0.052\end{array}$

Painful conditions (ref: $3-5$ )

$0-1$

$\begin{array}{llll}1.81 & 1.42 & 2.30 & <0.001 \\ 1.70 & 1.36 & 2.14 & <0.001\end{array}$

2

Average pain intensity (ref: $\geq 5$ )

$\begin{array}{lllll}\leq 2 & 0.98 & 0.76 & 1.26 & 0.880 \\ 3-4 & 1.02 & 0.83 & 1.25 & 0.872\end{array}$

Pain medications (ref: daily use)

$\begin{array}{lllll}\text { Never use } & 2.11 & 1.73 & 2.57<0.001\end{array}$

$\begin{array}{lllll}\text { Sometimes use } & 1.51 & 1.21 & 1.88 & <0.001\end{array}$

Nonprescription opioid use (ref: yes)

\begin{tabular}{lllll} 
No & 1.62 & 0.94 & 2.78 & 0.081 \\
\hline
\end{tabular}

Significantly higher earnings were reported by those with 0-5 painful days $(\$ 11,393)$ compared with those who had more than 20 painful days. Participants with 0-1 painful conditions reported $\$ 21,399$ greater earnings and those with 2 painful conditions reported $\$ 21,301$ greater earnings than those with 3 or more painful conditions. Those with no use of prescription medication to treat pain
Table 3 Demographic, injury, educational, and pain-related predictors of conditional hours worked $(n=1169)$

\begin{tabular}{rlr} 
& & \\
$\mathrm{dy} / \mathrm{dx}$ & $95 \%$ & $p$-value \\
& confidence & \\
& interval & \\
\hline
\end{tabular}

Injury severity (ref: $\mathrm{C} 1-\mathrm{C} 4$, nonambulatory)

$\begin{array}{lrrrr}\text { C5-C8, nonambulatory } & 3.99 & 2.27 & 5.71 & <0.001 \\ \text { Noncervical, nonambulatory } & 6.30 & 4.65 & 7.94 & <0.001 \\ \text { Ambulatory } & 11.03 & 9.43 & 12.64 & <0.001 \\ \text { Sex (ref: Female) } & & & & \\ \text { Male } & 4.08 & 3.31 & 4.86 & <0.001\end{array}$

Marital status (ref: Divorced/widowed/separated)

$\begin{array}{lrrrr}\begin{array}{l}\text { Married/member of } \\ \text { unmarried couple }\end{array} & 3.14 & 2.15 & 4.14 & <0.001 \\ \text { Never married } & -0.91 & -2.10 & 0.29 & 0.138 \\ \begin{array}{l}\text { Race (ref: Non-Hispanic Black) } \\ \text { Non-Hispanic White }\end{array} & 0.38 & -0.97 & 1.73 & 0.585 \\ \text { Other } & -0.29 & -2.43 & 1.85 & 0.788\end{array}$

Age at onset (ref: $\geq 50$ )

$<30$

$\geq 30$ and $<40$

$\geq 40$ and $<50$

$\begin{array}{llll}4.59 & 3.57 \quad 5.60 & <0.001\end{array}$

$3.64 \quad 2.40 \quad 4.88<0.001$

$\begin{array}{lllll}5.73 & 4.35 & 7.11<0.001\end{array}$

Time since onset (ref: $<10$ )

$\begin{array}{lllll}\geq 10 \text { and } \leq 19 & 1.43 & 0.55 & 2.30 & 0.001\end{array}$

$\geq 20$

Education (ref: $\leq$ High School)

2-year degree/trade school $\quad \begin{array}{lllll}1.33 & 0.40 & 2.25 & 0.005\end{array}$

4-year degree

$\begin{array}{llll}2.47 & 1.52 & 3.42<0.001\end{array}$

Postgraduate

$\begin{array}{llll}4.29 & 3.16 & 5.42<0.001\end{array}$

Painful days (ref: $>20$ pain days)

$\begin{array}{lllll}0-5 & 0.85 & -0.36 & 2.07 & 0.168\end{array}$

$\begin{array}{lllll}6-20 & -0.64 & -1.84 & 0.57 & 0.301\end{array}$

Painful conditions (ref: 3-5)

$\begin{array}{lllll}0-1 & -0.81 & -1.95 & 0.33 & 0.165\end{array}$

$\begin{array}{llllll}2 & 0.39 & -0.72 & 1.50 & 0.492\end{array}$

Average pain intensity (ref: $\geq 5$ )

$\begin{array}{lllll}\leq 2 & 1.23 & 0.13 & 2.33 & 0.029\end{array}$

$\begin{array}{lllll}3-4 & -1.27 & -2.21 & -0.33 & 0.008\end{array}$

Pain medications (ref: Daily use)

$\begin{array}{lllll}\text { Never use } & 3.14 & 2.20 & 4.09 & <0.001\end{array}$

$\begin{array}{lllll}\text { Sometimes use } & 1.86 & 0.75 & 2.98 & 0.001\end{array}$

Nonprescription opioid use (ref: yes)

\begin{tabular}{lllll} 
No & 4.87 & 2.08 & 7.66 & 0.001 \\
\hline
\end{tabular}

reported $\$ 23,916$ greater unconditional earnings, and those who occasionally used prescription medication to treat pain reported $\$ 10,063$ greater earnings than those who used prescription pain medication daily. Pain intensity and the use of nonprescription opioids were not significantly related to unconditional earnings. 
Table 4 Demographic, injury, educational, and pain-related predictors of unconditional hours worked $(n=4342)$

\begin{tabular}{lll}
\hline $\mathrm{dy} / \mathrm{dx}$ & $95 \%$ & $p$-value \\
& $\begin{array}{l}\text { confidence } \\
\text { interval }\end{array}$ \\
\hline
\end{tabular}

Injury severity (ref: $\mathrm{C} 1-\mathrm{C} 4$, nonambulatory)

$\begin{array}{lrrrr}\text { C5-C8, nonambulatory } & 2.29 & 1.02 & 3.56 & <0.001 \\ \text { Noncervical, nonambulatory } & 4.30 & 3.04 & 5.56 & <0.001 \\ \text { Ambulatory } & 10.75 & 9.47 & 12.04 & <0.001 \\ \text { Sex (ref: Female) } & & & & \\ \quad \text { Male } & 1.73 & 0.80 & 2.65 & <0.001\end{array}$

Marital status (ref: Divorced/widowed/separated)

Married/member of

$\begin{array}{rrrr}3.30 & 2.15 & 4.44 & <0.001 \\ 0.04 & -1.32 & 1.39 & 0.959 \\ 6.00 & 4.91 & 7.10 & <0.001 \\ 3.98 & 1.49 & 6.47 & 0.002\end{array}$

Never married

Race (ref: Non-Hispanic Black)

Non-Hispanic White

Other

Age at onset (ref: $\geq 50$ )

$<30$

$\geq 30$ and $<40$

$\geq 40$ and $<50$

Time since onset (ref: $<10)$

$\geq 10$ and $\leq 19$

$\geq 20$

Education (ref: $\leq$ High School)

2-year degree/trade school

4-year degree

Postgraduate

Painful days (ref: $>20$ pain days)

$$
\begin{aligned}
& 0-5 \\
& 6-20 \\
& \text { Painful conditions (ref: } 3-5 \text { ) } \\
& 0-1 \\
& 2 \\
& \text { Average pain intensity (ref: } \geq 5 \text { ) } \\
& \leq 2 \\
& 3-4
\end{aligned}
$$

Pain medications (ref: Daily use)

\begin{tabular}{lllll} 
Never use & 5.68 & 4.46 & 6.91 & $<0.001$ \\
Sometimes use & 3.25 & 1.92 & 4.57 & $<0.001$ \\
$\begin{array}{l}\text { Nonprescription opioid use (ref: yes) } \\
\text { No }\end{array}$ & 3.53 & 1.17 & 5.90 & 0.003 \\
\hline
\end{tabular}

\section{Discussion}

The unique contributions of this study include: (1) identification of multiple pain and medication use indicators with

\begin{tabular}{|c|c|c|c|c|}
\hline C5-C8, nonambulatory & 9088 & -4914 & 23090 & 0.203 \\
\hline $\begin{array}{l}\text { Noncervical, } \\
\text { nonambulatory }\end{array}$ & 14329 & 872 & 27785 & 0.037 \\
\hline Ambulatory & 23080 & 9930 & 36231 & 0.00 \\
\hline \multicolumn{5}{|l|}{ Sex (ref: Female) } \\
\hline Male & 19238 & 13610 & 24865 & $<0.00$ \\
\hline
\end{tabular}
employment, (2) multiple indicators of quality employment, and (3) utilization of econometric modeling quantifying conditional and unconditional hours and earnings outcomes.
Table 5 Demographic, injury, educational, and pain-related predictors of conditional earnings $(n=1170)$

$$
\begin{array}{ll}
\text { Coefficient } & \begin{array}{l}
95 \% \text { confidence } \\
\text { interval }
\end{array}
\end{array}
$$

Injury severity (ref: $\mathrm{C} 1-\mathrm{C} 4$, nonambulatory)

Marital status (ref: Divorced/widowed/separated)

Married/member of $\quad 7481 \quad 570-14392$ unmarried couple

$\begin{array}{lllll}\text { Never married } & -13241 & -21459 & -5023 & 0.002\end{array}$

Race (ref: Non-Hispanic Black)

Non-Hispanic White $\quad 17042 \quad 7317 \quad 26766 \quad 0.001$

$\begin{array}{lllll}\text { Other } & 12411 & -2579 & 27400 & 0.105\end{array}$

Age at onset (ref: $\geq 50$ )

$\begin{array}{llllr}<30 & -821 & -7743 & 6101 & 0.816 \\ \geq 30 \text { and }<40 & 6576 & -1462 & 14613 & 0.109 \\ \geq 40 \text { and }<50 & 16429 & 7719 & 25139 & <0.001 \\ \text { Time since onset }(\text { ref: }<10) & & & & \\ \geq 10 \text { and } \leq 19 & 4794 & -1483 & 10982 & 0.135 \\ \geq 20 & 15269 & 8908 & 21629 & <0.001\end{array}$

Education (ref: $\leq$ High School)

2-year degree/trade school $9177 \quad 2550 \quad 15805 \quad 0.007$

4-year degree $\quad 32774 \quad 26045 \quad 39504<0.001$

Postgraduate $\quad 42420 \quad 34575 \quad 50264<0.001$

Painful days (ref: $>20$ pain days)

$\begin{array}{lllll}0-5 & 4856 & -3584 & 13296 & 0.259 \\ 6-20 & -1896 & 10225 & 6434 & 0.656\end{array}$

Painful conditions (ref: $3-5$ )

$\begin{array}{lllll}0-1 & 3817 & -4096 & 11729 & 0.344\end{array}$

$\begin{array}{lllll}2 & 5373 & -2334 & 13080 & 0.172\end{array}$

Average pain intensity (ref: $\geq 5$ )

\begin{tabular}{lrrrr}
$\leq 2$ & 1420 & -6220 & 9059 & 0.716 \\
$3-4$ & -445 & -7028 & 6138 & 0.895 \\
$\begin{array}{l}\text { Pain medications } \\
\text { Never use }\end{array}$ & & & \\
Nef: Daily use) & 2218 & -4341 & 8777 & 0.507 \\
Sometimes use & -2527 & -9944 & 4890 & 0.504 \\
$\begin{array}{l}\text { Nonprescription opioid use (ref: yes) } \\
\text { No }\end{array}$ & & & \\
\hline
\end{tabular}

The primary findings were that pain and pain medication use, including use of nonprescription opioids, are important covariates of quality employment among those with SCI. However, not all of the pain indicators were consistently related to employment outcomes, so it is important to consider the specific employment outcome and pain 
Table 6 Demographic, injury, educational, and pain-related predictors of unconditional earnings $(n=4255)$

Coefficient $95 \%$ confidence $p$-value interval

Injury severity (ref: $\mathrm{C} 1-\mathrm{C} 4$, nonambulatory)

\begin{tabular}{|c|c|c|c|c|}
\hline $\mathrm{C} 5-\mathrm{C} 8$, nonambulatory & 22970 & 7916 & 38024 & 0.003 \\
\hline $\begin{array}{l}\text { Noncervical, } \\
\text { nonambulatory }\end{array}$ & 33074 & 18615 & 47532 & $<0.001$ \\
\hline Ambulatory & 64995 & 50519 & 79470 & $<0.001$ \\
\hline \multicolumn{5}{|l|}{ Sex (ref: Female) } \\
\hline Male & 16559 & 9868 & 23250 & $<0.001$ \\
\hline \multicolumn{5}{|c|}{ Marital status (ref: Divorced/widowed/separated) } \\
\hline $\begin{array}{l}\text { Married/member of } \\
\text { unmarried couple }\end{array}$ & 17858 & 10086 & 25629 & $<0.001$ \\
\hline Never married & -8392 & -17724 & 941 & 0.078 \\
\hline \multicolumn{5}{|c|}{ Race (ref: Non-Hispanic Black) } \\
\hline Non-Hispanic White & 47258 & 37145 & 57370 & $<0.001$ \\
\hline Other & 28997 & 12871 & 45122 & $<0.001$ \\
\hline \multicolumn{5}{|l|}{ Age at onset (ref: $\geq 50$ ) } \\
\hline$<30$ & 35089 & 26550 & 43629 & $<0.001$ \\
\hline$\geq 30$ and $<40$ & 36930 & 27358 & 46503 & $<0.001$ \\
\hline$\geq 40$ and $<50$ & 34803 & 24875 & 44732 & $<0.001$ \\
\hline \multicolumn{5}{|l|}{ Time since onset (ref: $<10$ ) } \\
\hline$\geq 10$ and $\leq 19$ & 9496 & 2030 & 16962 & 0.013 \\
\hline$\geq 20$ & 17913 & 10629 & 25197 & $<0.001$ \\
\hline \multicolumn{5}{|l|}{ Education (ref: $\leq$ High School) } \\
\hline 2-year degree/trade school & 24545 & 17040 & 32050 & $<0.001$ \\
\hline 4-year degree & 57308 & 49150 & 65466 & $<0.001$ \\
\hline Postgraduate & 64758 & 55176 & 74339 & $<0.001$ \\
\hline \multicolumn{5}{|c|}{ Painful days (ref: >20 pain days) } \\
\hline $0-5$ & 11393 & 1711 & 21075 & 0.021 \\
\hline $6-20$ & 6374 & -2909 & 15656 & 0.178 \\
\hline \multicolumn{5}{|l|}{ Painful conditions (ref: $3-5$ ) } \\
\hline $0-1$ & 21399 & 12171 & 30626 & $<0.001$ \\
\hline 2 & 21301 & 12503 & 30100 & $<0.001$ \\
\hline \multicolumn{5}{|c|}{ Average pain intensity (ref: $\geq 5$ ) } \\
\hline$\leq 2$ & 542 & -8671 & 9755 & 0.908 \\
\hline $3-4$ & -1083 & -8816 & 6650 & 0.784 \\
\hline \multicolumn{5}{|c|}{ Pain medications (ref: Daily use) } \\
\hline Never use & 23916 & 16123 & 31708 & $<0.001$ \\
\hline Sometimes use & 10063 & 1566 & 18560 & 0.020 \\
\hline \multicolumn{5}{|c|}{ Nonprescription opioid use (ref: yes) } \\
\hline No & 12054 & -8619 & 32726 & 0.253 \\
\hline
\end{tabular}

indicator when evaluating the potential risks of pain and pain medication with quality employment.

The frequency of treatment of pain with prescription medication was the pain parameter most consistently related to employment outcomes, as participants who did not use prescription medication for pain had over double the odds of employment compared with daily users, as well as higher conditional and unconditional hours per week and higher unconditional earnings. However, it was not related to conditional earnings. Use of nonprescription opioids was associated with fewer conditional and unconditional work hours but not related to earnings. Taken together, it appears that use of prescription pain medications is strongly associated with the diminished ability to obtain and retain employment and to maintain a greater number of work hours; but less strongly related to another quality indicator-conditional earnings. Vocational rehabilitation professionals should routinely screen for prescription use of opioids as a barrier to obtaining and maintaining employment, as well as quality indicators of work intensity (i.e., hours) and earnings.

Of the nonpharmacological pain indicators, the number of painful conditions and the number of days adversely affected by pain were highly correlated with employment outcomes, whereas average pain severity was only related to number of hours working. Overall, this suggests the potential debilitating aspects of painful conditions and adverse effect of pain on employment. It also creates a dilemma for healthcare providers. The presence of multiple painful conditions, leading to days adversely affected by pain, is associated with both lower employment rates and poor quality outcomes. Therefore, healthcare providers should seek effective treatments for pain management. However, those who are treated daily with prescription medications have lower likelihood of employment and work fewer hours per week.

The relationships between demographic, injury, and educational variables with employment outcomes are consistent with previous research $[21,35,36]$ and do not require a great deal of elaboration. It is important to note, however, the highly significant associations between injury severity and years of education with multiple employment outcomes. For each of these variables, lower odds of employment were compounded by fewer hours worked and lower earnings. It is also important to note sex differences in quality outcomes, both hours per week working and earnings, even though no differences were observed in the odds of employment. For race/ethnicity, the very large disparities in the odds of employment for non-Hispanic Blacks are particularly of current concern and are compounded by lower conditional earnings. Therefore, non-Hispanic Blacks who are working similar hours as non-Hispanic Whites have lower earnings.

The overall findings have several important implications for clinical and vocational practice. First, the highly significant relationships between multiple pain parameters and quality employment outcomes underscore the interrelationships between health and function and employment. The development of successful interventions for pain may also favorably impact the probability of obtaining and retaining employment and also work intensity (i.e., hours working) and earnings. Second, beyond the effects of the painful conditions themselves, the clear and present relationships with the use of 
prescribed and nonprescribed opioids with employment outcomes raises significant concerns. Even if pharmacological interventions are successful in reducing pain, they may adversely affect the ability to obtain and maintain quality employment. Third, the persistent disparities in employment outcomes as a function of injury severity, race/ethnicity, and sex draw attention to the need to work to eliminate disparities in outcomes. Given the overwhelming differences in quality employment related to educational milestones, formal education appears to be the key to promoting employment outcomes.

\section{Study strengths and limitations}

There were several important methodologic considerations. First, all data were self-report, highlighting the importance of accuracy of reporting. This may affect the reporting of sensitive information, such as use of nonprescription opioids. Second, all data were cross-sectional and were collected over an approximate 5-year time period (2012-2016). Therefore, we cannot infer causality. The findings do however present a pattern of interrelationships that require strategies for intervention. Third, our direct measurement of opioid use is limited to nonprescribed use, whereas prescription medication to treat pain represents a proxy for opioid use. Use of nonprescription opioids was measured by a single item with the ASSIST; therefore, we cannot determine what substance the individual is using, and different substances within this category may have differing relationships with employment. In addition, the data were taken from a larger study on health outcomes, rather than exclusively focused on pain, so measurement of employment outcomes is limited to those variables utilized in the analysis. We also cannot differentiate nociceptive and neuropathic pain. Fourth, although there are advantages with population-based cohorts, enrollment is more difficult than with clinical cohorts, so response rates are typically lower. This may affect absolute estimates of employment rates but would be less likely to affect the interrelationships between the predictor variables and outcomes. Fifth, there are limits to generalizability, particularly absolute estimates of employment rates and other quality indicators of employment, based on utilization of cohorts from two geographic regions within the United States. Lastly, we recoded response categories to a more manageable and limited number of different groups for the econometric analyses. Some of the distinctions were rather arbitrary.

\section{Future research}

Additional research is needed utilizing more detailed assessments of pain, specific pain medications, and additional employment outcomes. Future research should focus on different types of opioid use, including specific substances. It would also benefit from alternative or additional data sources that may measure opioid use more objectively, as the measures used in the current study are general in nature and all based on self-report. Furthermore, longitudinal research is needed to identify these relationships over time, as that would provide more evidence related to causality. Intervention studies are needed to better identify how alternative treatments for pain may also affect vocational outcomes.

\section{Conclusion}

Pain and pharmacological treatment of pain are related to employment outcomes, with the specific relationships dependent on the outcome. Disparities in employment rates, which reflect ability to obtain and sustain employment, are compounded by additional disparities in quality of employment for those who have more severe SCI, are non-Hispanic Black, and have lower levels of education.

\section{Data availability}

The datasets generated and/or analyzed during the current study are not publicly available due to the privacy concerns of study participants and are not standardized to be in a publicly interpretable format.

Author contributions JK was the principal investigator and was responsible for the research design and contributed to the introduction, discussion, and overall development of the manuscript. CDG was responsible for the analyses and research methods section and contributed to the results and conclusion sections. KR was responsible for the literature search, introduction, and editing of the manuscript. CL contributed to the analyses and research methods sections.

Funding The contents of this publication were developed under grants from the National Institute on Disability, Independent Living, and Rehabilitation Research (NIDILRR grant numbers 90DP0050 and 90RT5003) and the South Carolina Spinal Cord Injury Research Fund (SCIRF \#2017 SI-02). NIDILRR is a Center within the Administration for Community Living (ACL), Department of Health and Human Services (HHS). The contents of this publication do not necessarily represent the policy of NIDILRR, ACL, HHS, or the SCRIF and you should not assume endorsement by the Federal Government or the state of South Carolina.

\section{Compliance with ethical standards}

Conflict of interest The authors declare that they have no conflict of interest. 
Ethical statement We certify that all applicable institutional and governmental regulations concerning the ethical use of human volunteers were followed during the course of this research.

Publisher's note Springer Nature remains neutral with regard to jurisdictional claims in published maps and institutional affiliations.

\section{References}

1. Hammell KW, Miller WC, Forwell SJ, Forman BE, Jacobsen BA. Fatigue and spinal cord injury: a qualitative analysis. Spinal Cord. 2009;47:44-9.

2. Krause JS, DeVivo MJ, Jackson AB. Health status, community integration, and economic risk factors for mortality after spinal cord injury. Arch Phys Med Rehabil. 2004;85:1764-73.

3. Leduc BE, Lepage Y. Health-related quality of life after spinal cord injury. Disabil Rehabil. 2002;24:196-202.

4. Lidal IB, Veenstra M, Hjeltnes N, Biering-Sorensen F. Healthrelated quality of life in persons with long-standing spinal cord injury. Spinal Cord. 2008;46:710-5.

5. Trenaman LM, Miller WC, Escorpizo R, the SRT. Interventions for improving employment outcomes among individuals with spinal cord injury: a systematic review. Spinal Cord. 2014;52:799-4.

6. Young AE, Murphy GC. Employment status after spinal cord injury (1992-2005): a review with implications for interpretation, evaluation, further research, and clinical practice. Int J Rehabil Res. 2009;32:1-11.

7. Hilton G, Unsworth C, Murphy G The experience of attempting to return to work following spinal cord injury: a systematic review of the qualitative literature. Disabil Rehabil. 2017;40:1-9.

8. Krause JS, Terza JV, Dismuke C. Factors associated with labor force participation after spinal cord injury. J Voc Rehab. 2010;33:89-99.

9. Krause JS, Terza JV, Saunders LL, Dismuke CE. Delayed entry into employment after spinal cord injury: factors related to time to first job. Spinal Cord. 2010;48:487-91.

10. Krause JS, Edles P, Charlifue S. Changes in employment status and earnings after spinal cord injury: a pilot comparison from pre to post injury. Top Spinal Cord Inj Rehabil. 2011;16:74-9.

11. Wehman P, Wilson K, Parent W, Sherron-Targett P, McKinley W. Employment satisfaction of individuals with spinal cord injury. Am J Phys Med Rehabil. 2000;79:161-9.

12. Meade MA, Barrett K, Ellenbogen PS, Jackson MN. Work intensity and variations in health and personal characteristics of individuals with spinal cord injury. J Voc Rehab. 2006;25:13-9.

13. Krause JS, Saunders LL, Acuna J. Gainful employment and risk of mortality after spinal cord injury: effects beyond that of demographic, injury and socioeconomic factors. Spinal Cord. 2012;50:784-8.

14. Krause JS, Saunders LL. Socioeconomic and behavioral risk factors for mortality: do risk factors observed after spinal cord injury parallel those from the general USA population? Spinal Cord. 2012;50:609-13.

15. Finnerup NB. Neuropathic pain and spasticity: intricate consequences of spinal cord injury. Spinal Cord. 2017;55:1046-50.

16. Hagen EM, Rekand T. Management of neuropathic pain associated with spinal cord injury. Pain Ther. 2015;4:51-65.

17. Andresen SR, Biering-Sorensen F, Hagen EM, Nielsen JF, Bach FW, Finnerup NB. Pain, spasticity and quality of life in individuals with traumatic spinal cord injury in Denmark. Spinal Cord. 2016;54:973-9.
18. Dijkers M, Bryce T, Zanca J. Prevalence of chronic pain after traumatic spinal cord injury: a systematic review. J Rehabil Res Dev. 2009;46:13-29.

19. Marti A, Boes S, Lay V, Reuben Escorpizo PT, Trezzini B. The association between chronological age, age at injury and employment: Is there a mediating effect of secondary health conditions? Spinal Cord. 2016;54:239-44.

20. Widerstrom-Noga EG, Felipe-Cuervo E, Yezierski RP. Chronic pain after spinal injury: interference with sleep and daily activities. Arch Phys Med Rehabil. 2001;82:1571-7.

21. Ottomanelli L, Lind L. Review of critical factors related to employment after spinal cord injury: implications for research and vocational services. J Spinal Cord Med. 2009;32:503-31.

22. Mann R, Schaefer C, Sadosky A, Bergstrom F, Baik R, Parsons B, et al. Burden of spinal cord injury-related neuropathic pain in the United States: retrospective chart review and cross-sectional survey. Spinal Cord. 2013;51:564-70.

23. Norrbrink $C$, Lundeberg $T$. Tramadol in neuropathic pain after spinal cord injury: a randomized, double-blind, placebo-controlled trial. Clin J Pain. 2009;25:177-84.

24. Kitzman P, Cecil D, Kolpek JH. The risks of polypharmacy following spinal cord injury. J Spinal Cord Med. 2017;40:147-53.

25. Clark JM, Cao Y, Krause JS. Risk of pain medication misuse after spinal cord injury: the role of substance use, personality, and depression. J Pain. 2017;18:166-77.

26. Krause JS, Clark JM, Saunders LL. Pain medication misuse among participants with spinal cord injury. Spinal Cord. 2015;53:630-5.

27. Butler J, Langlois JA. Central nervous system injury surveillance: annual data submission standards-2000. Atlanta, GA: US Department of Health and Human Services, Centers for Disease Control and Prevention, National Center for Injury Prevention and Control; 2001.

28. CDC. Behavioral risk factor surveillance system. 2010. http://www.cdc.gov/brfss/questionnaires/pdf-ques/2010brfss.pdf.

29. Adams LL, Gatchel RJ, Robinson RC, Polatin P, Gajraj N, Deschner $\mathrm{M}$, et al. Development of a self-report screening instrument for assessing potential opioid medication misuse in chronic pain patients. J Pain Symptom Manag. 2004;27:440-59.

30. Holmes CP, Gatchel RJ, Adams LL, Stowell AW, Hatten A, Noe C, et al. An opioid screening instrument: long-term evaluation of the utility of the pain medication questionnaire. Pain Pr. 2006;6:74-88.

31. Cleeland CS, Ryan KM. Pain assessment: global use of the brief pain inventory. Ann Acad Med Singap. 1994;23:129-38.

32. Humeniuk R, Ali R, Babor TF, Farrell M, Formigoni ML, Jittiwutikarn J, et al. Validation of the alcohol, smoking and substance involvement screening test (ASSIST). Addiction. 2008; 103:1039-47.

33. Humeniuk R, Henry-Edwards S, Ali R, Poznyak V, Monteiro M. The alcohol smoking and substance involvement screening test (ASSIST): manual for use in primary care. 2010. http://www.who. int/substance_abuse/activities/en/Draft_The_ASSIST_Guidelines. pdf.

34. Belotti F, Deb P, Manning WG, Norton EC. twopm: two-part models. Stata J. 2015;15:3-20.

35. Krause JS, Reed KS. Obtaining employment after spinal cord injury: Relationship with pre- and post-injury education. Rehabil Couns Bull. 2009;53:27-33.

36. Hirsh AT, Molton IR, Johnson KL, Bombardier CH, Jensen MP. The relationship of chronological age, age at injury, and duration of injury to employment status in individuals with spinal cord injury. Psychol Inj Law. 2009;2:263-75. 